\title{
Cytoreductive nephrectomy following treatment with anti-vascular endothelial growth factor-targeted agents: is it surgically safe?
}

\author{
Hideaki Miyake \\ Department of Urology, Hamamatsu University School of Medicine, Hamamatsu, Japan \\ Correspondence to: Dr. Hideaki Miyake. Department of Urology, Hamamatsu University School of Medicine, Hamamatsu 431-3192, Japan. Email: \\ hideakimiyake@hotmail.com. \\ Provenance: This is an invited article commissioned by the Section Editor Dr. Xiao Li (Department of Urology, Jiangsu Cancer Hospital, Jiangsu \\ Institute of Cancer Research, Nanjing Medical University Affiliated Cancer Hospital, Nanjing, China). \\ Comment on: De Bruijn RE, Mulders P, Jewett MA, et al. Surgical Safety of Cytoreductive Nephrectomy Following Sunitinib: Results from the \\ Multicentre, Randomised Controlled Trial of Immediate Versus Deferred Nephrectomy (SURTIME). Eur Urol 2019;76:437-40.
}

Submitted Sep 04, 2019. Accepted for publication Sep 17, 2019.

doi: 10.21037/atm.2019.09.106

View this article at: http://dx.doi.org/10.21037/atm.2019.09.106

In the era of cytokine therapy, 2 prospective randomized clinical trials (RCTs) demonstrated significantly more superior overall survival (OS) of metastatic renal cell carcinoma (mRCC) patients receiving cytoreductive nephrectomy followed by interferon- $\alpha($ IFN- $\alpha$ ) than of those treated with IFN- $\alpha$ alone $(1,2)$; therefore, it has been recognized as a standard therapeutic approach for mRCC patients to perform cytoreductive nephrectomy prior to the introduction of systemic therapy.

During the last decade, various types of novel moleculartargeted agent have been approved for the treatment of patients with mRCC, and these agents, particularly antivascular endothelial growth factor (VEGF)-targeted agents, have been widely introduced in real-world clinical practice, resulting in marked changes in the multimodal therapeutic strategy against mRCC (3). In the era of targeted agents as well, despite being retrospectively conducted, a number of studies revealed the prognostic benefit of immediate surgical resection combined with targeted therapy for mRCC patients compared with targeted therapy alone (4-6). For example, Heng et al. reported the significantly beneficial impact of cytoreductive nephrectomy on OS in synchronous mRCC patients treated with targeted agents compared with those receiving targeted therapy alone, even after adjusting for prognostic factors, based on data from the International Metastatic Renal Cell Carcinoma Database Consortium (4). Accordingly, nephrectomy has continued to be performed based on the assumption that the therapeutic benefit was not changed after the introduction of targeted agents.
Recently, Méjean et al. conducted the CARMENA trial, which compared the outcomes of cytoreductive nephrectomy followed by sunitinib versus sunitinib alone in mRCC patients classified into an intermediate or a poor risk group according to the Memorial Sloan Kettering Cancer Center (MSKCC) prognostic model. The median OS was 13.9 and 18.4 months in the nephrectomy-sunitinib and sunitinib alone groups, respectively, and the hazard ratio for death on analysis of OS was 0.89 . Thus, this study demonstrated the noninferiority of sunitinib alone compared with nephrectomy-sunitinib regarding OS (7). Although various important limitations associated with the CARMENA trial were pointed out, this was the first RCT suggesting the prognostic benefit of avoiding surgery for selected mRCC patients.

In agreement with the CARMENA trial, the outcomes of the SURTIME trial were recently reported (8). This trial included mRCC patients who were randomized into immediate cytoreductive nephrectomy followed by sunitinib therapy or treatment with 3 cycles of sunitinib followed by cytoreductive nephrectomy in order to assess whether pretreatment with sunitinib prior to planned nephrectomy improves prognostic outcomes by identifying patients who are not likely to benefit from cytoreductive nephrectomy. In this trial, the 28 -week progression-free rate were $42 \%$ and $43 \%$ in the immediate and deferred cytoreductive nephrectomy groups, respectively, and the hazard ratio of OS between these 2 groups was 0.57 with median OSs of 15.0 and 32.4 months in the immediate and deferred 
Table 1 Comparison of surgery-related adverse events (AEs) in the SURTIME trial

\begin{tabular}{lcc}
\hline Adverse events & Immediate surgery group $(\mathrm{n}=46)$ & Deferred surgery group $(\mathrm{n}=40)$ \\
\hline Perioperative AEs $(\%)^{*}$ & $24(52.2)$ & $18(52.9)$ \\
Intraoperative AEs $(\%)^{*}$ & $14(30.4)$ & $6(17.6)$ \\
Postoperative AEs (\%)* & $18(39.1)$ & $16(47.1)$ \\
Postoperative grade 3-4 AEs (\%) & $8(17.4)$ & $7(17.5)$ \\
Readmission within 30 days after surgery (\%) & $4(8.7)$ & $2(5.0)$ \\
In-hospital mortality (\%) & $1(2.2)$ & $1(2.5)$ \\
\hline
\end{tabular}

${ }^{*}$, calculated based on the number of patients undergoing treatments according to per protocol.

cytoreductive nephrectomy groups, respectively. These findings suggest that a deferred approach to cytoreductive nephrectomy considering the response to sunitinib therapy may be superior to conducting cytoreductive nephrectomy first followed by the introduction of sunitinib. However, similar to the CARMENA trial, the SURTIME trial had several limitations. For example, this study was underpowered for the assessment of the prognostic endpoint due to insufficient recruitment, and because of poor accrual, the 28-week progression-free rate was set as a primary endpoint, instead of progression-free survival.

The findings of these 2 RCTs suggest that immediate cytoreductive nephrectomy may not result in an evident additional benefit compared with a deferred surgical approach. In fact, the consideration of cytoreductive nephrectomy is currently recommended in patients showing a response to initial treatment with anti-VEGFtargeted agents by the European Association of Urology guidelines (9). However, there is concern over the surgical safety of cytoreductive nephrectomy following treatment with anti-VEGF-targeted agents, which still may affect the prevalence of deferred cytoreductive nephrectomy in realworld clinical practice. In several previous retrospective studies, mRCC patients treated with deferred cytoreductive nephrectomy were reported to show increased surgeryrelated adverse events (AEs) following presurgical antiVEGF-targeted therapy when compared with those receiving immediate surgery (10-12). For example, Margulis et al. analyzed perioperative complications in 44 patients receiving targeted agents prior to cytoreductive nephrectomy as well as a matched cohort of 58 patients undergoing up-front nephrectomy, and reported that a total of 39 complications occurred in 17 (39\%) patients treated with presurgical targeted therapy and in 16 (28\%) undergoing up-front resection (10).
Recently, De Bruijn et al. reported the detailed findings of the SURTIME trial focusing on the surgical safety of cytoreductive nephrectomy, one of the prespecified secondary endpoints in this trial (13). Table 1 summarizes the comparison of surgery-related AEs between the immediate and deferred cytoreductive nephrectomy groups. In this trial, surgery-related AEs developed in $52 \%$ and $53 \%$ of patients undergoing cytoreductive nephrectomy in the immediate and deferred settings, respectively, while the proportion of patients with intraoperative surgery-related AEs was higher in the immediate surgery group. In addition, there were no significant differences in the incidences of postoperative AEs corresponding to grade 3 or 4 , readmission within 30 days after cytoreductive nephrectomy or in-hospital mortality between the two groups. These outcomes with a lower incidence of intraoperative AEs in the deferred cytoreductive nephrectomy group were contrary to those in previous retrospective studies reporting higher postoperative complication rates associated with intraoperative adhesions, difficult tissue dissection and wound healing problems in patients treated with presurgical anti-VEGF-targeted therapy. In addition to retrospective studies, there have been some prospective studies evaluating the impact of presurgical therapy with anti-VEGF-targeted agents on mRCC patients $(14,15)$. AE profiles reported in these studies were similar to those in the deferred cytoreductive nephrectomy group in the SURTIME trial. In a combined assessment of 2 prospective studies of presurgical sunitinib, postoperative AEs were shown to occur in $27 \%$, including $11 \%$ with grade $3-5$ AEs (14), while $22 \%$ of patients who underwent cytoreductive nephrectomy experienced grade 3-5 AEs in a prospective study of presurgical pazopanib (15).

However, when interpreting the outcomes of surgeryrelated AEs in the SURTIME trial, it is important to be 
aware of the following issues: (I) the majority of included patients were classified into the MSKCC intermediate risk group. (II) Due to the inclusion criteria requiring 3 or fewer surgical risk factors, including a grade 2 or higher level of serum albumin, serum lactate dehydrogenase level greater than 1.5 times the upper limit of normal, liver metastases, symptoms caused by metastases, retroperitoneal or supradiaphragmatic lymph node metastases and stage cT3 or T4 disease, this study was composed of patients with favorable surgical risk factors alone.

Although the significance of the SURTIME trial is still being discussed, it provides the only published randomized findings on the sequence of cytoreductive nephrectomy and anti-VEGF-targeted therapy (8). With respect to the $\mathrm{AE}$ profiles in this trial, higher intraoperative and similar postoperative complication rates in the immediate surgery group compared with the deferred surgery group were noted. Accordingly, surgery even after sunitinib therapy was comprehensively shown to be safely performed in this trial (13), suggesting that a deferred approach may improve cancer-associated morbidity by reducing the tumor size and neovascularization, which may also lower risks associated with surgery. Although it is important to carefully recognize surgery-related AEs caused by presurgical treatment with anti-VEGF-targeted agents, sub-analyses of the SURTIME study focusing on surgery-related AEs indicate that mRCC patients may not have surgical complications irrespective of whether they receive treatment with sunitinib before or after cytoreductive nephrectomy.

\section{Acknowledgments}

None.

\section{Footnote}

Conflicts of Interest: The author has no conflicts of interest to declare.

Ethical Statement: The author is accountable for all aspects of the work in ensuring that questions related to the accuracy or integrity of any part of the work are appropriately investigated and resolved.

\section{References}

1. Flanigan RC, Salmon SE, Blumenstein BA, et al. Nephrectomy followed by interferon alfa-2b compared with interferon alfa-2b alone for metastatic renal-cell cancer. N Engl J Med 2001;345:1655-9.

2. Mickisch GH, Garin A, van Poppel H, et al. Radical nephrectomy plus interferon- $\alpha$-based immunotherapy compared with interferon $\alpha$ alone in metastatic renal-cell carcinoma: a randomised trial. Lancet 2001;358:966-70.

3. Bedke J, Gauler T, Grünwald V, et al. Systemic therapy in metastatic renal cell carcinoma. World J Urol 2017;35:179-88.

4. Heng DY, Wells JC, Rini BI, et al. Cytoreductive nephrectomy in patients with synchronous metastases from renal cell carcinoma: results from the International Metastatic Renal Cell Carcinoma Database Consortium. Eur Urol 2014;66:704-10.

5. Bamias A, Tzannis K, Papatsoris A, et al. Prognostic significance of cytoreductive nephrectomy in patients with synchronous metastases from renal cell carcinoma treated with first-line sunitinib: a European multiinstitutional study. Clin Genitourin Cancer 2014;12:373-83.

6. Miyake H, Sugiyama T, Aki R, et al. Oncological outcomes after cytoreductive nephrectomy for patients with metastatic renal cell carcinoma with inferior vena caval tumor thrombus. Int J Clin Oncol 2018;23:553-8.

7. Méjean A, Ravaud A, Thezenas S, et al. Sunitinib alone or after nephrectomy in metastatic renal-cell carcinoma. $\mathrm{N}$ Engl J Med 2018;379:417-27.

8. Bex A, Mulders P, Jewett M, et al. Comparison of Immediate vs Deferred Cytoreductive Nephrectomy in Patients With Synchronous Metastatic Renal Cell Carcinoma Receiving Sunitinib: The SURTIME Randomized Clinical Trial. JAMA Oncol 2019;5:164-70.

9. Bex A, Albiges L, Ljungberg B, et al. Updated European Association of Urology Guidelines for Cytoreductive Nephrectomy in Patients with Synchronous Metastatic Clear-cell Renal Cell Carcinoma. Eur Urol 2018;74:805-9.

10. Margulis V, Matin SF, Tannir N, et al. Surgical morbidity associated with administration of targeted molecular therapies before cytoreductive nephrectomy or resection of locally recurrent renal cell carcinoma. J Urol 2008;180:94-8.

11. Thomas AA, Rini BI, Stephenson AJ, et al. Surgical resection of renal cell carcinoma after targeted therapy. J Urol 2009;182:881-6.

12. Shaw GL, Hussain M, Nair R, et al. Performing cytoreductive nephrectomy following targeted sunitinib therapy for metastatic renal cell carcinoma: a surgical perspective. Urol Int 2012;89:83-8.

13. De Bruijn RE, Mulders P, Jewett MA, et al. Surgical Safety 
of Cytoreductive Nephrectomy Following Sunitinib: Results from the Multicentre, Randomised Controlled Trial of Immediate Versus Deferred Nephrectomy (SURTIME). Eur Urol 2019;76:437-40.

14. Powles T, Kayani I, Blank C, et al. The safety and efficacy of sunitinib before planned nephrectomy in metastatic

Cite this article as: Miyake H. Cytoreductive nephrectomy following treatment with anti-vascular endothelial growth factor-targeted agents: is it surgically safe? Ann Transl Med 2019;7(Suppl 8):S347. doi: 10.21037/atm.2019.09.106 clear cell renal cancer. Ann Oncol 2011;22:1041-7.

15. Powles T, Sarwar N, Stockdale A, et al. Safety and Efficacy of Pazopanib Therapy Prior to Planned Nephrectomy in Metastatic Clear Cell Renal Cancer. JAMA Oncol 2016;2:1303-9. 